Applied Physiology, Nutrition, and Metabolism

Canadian Science Publishing Physiologie appliquée, nutrition et métabolisme

\title{
Short-term changes in daily movement behaviour influence salivary C-reactive protein in healthy women
}

\begin{tabular}{|r|l|}
\hline Journal: & Applied Physiology, Nutrition, and Metabolism \\
\hline Manuscript ID & apnm-2017-0758.R2 \\
\hline Manuscript Type: & Brief communication \\
\hline Complete List of Authors: & $\begin{array}{l}\text { Willoughby, Taura; University of Lethbridge, Kinesiology and Physical } \\
\text { Education } \\
\text { Doan, Jon; University of Lethbridge } \\
\text { Currie, Cheryl; University of Lethbridge } \\
\text { Copeland, Jennifer L.; University of Lethbridge, }\end{array}$ \\
\hline Keyword: & $\begin{array}{l}\text { sedentary, inflammation, physical activity < exercise, saliva, C reactive } \\
\text { protein }\end{array}$ \\
\hline $\begin{array}{r}\text { Is the invited manuscript for } \\
\text { consideration in a Special } \\
\text { Issue? : }\end{array}$ & N/A \\
\hline
\end{tabular}


3 Short-term changes in daily movement behaviour influence salivary C-reactive protein in 4 healthy women.

5

6

Taura N. Willoughby ${ }^{1}$, Jon Doan ${ }^{1}$, Cheryl L. Currie ${ }^{2}$, Jennifer L. Copeland ${ }^{1}$

\section{Corresponding author:}

${ }^{1}$ Department of Kinesiology \& Physical Education, University of Lethbridge

${ }^{2}$ Faculty of Health Sciences, University of Lethbridge

18 Department of Kinesiology \& Physical Education,

19 University of Lethbridge

204401 University Drive, Lethbridge, Alberta, Canada. T1K 3M4

21 Phone: (403) 317-2804 Fax: (403) 380-1839

22 jennifer.copeland@uleth.ca

23

24 
26 ABSTRACT

27 This study assessed the effect of changing daily movement behaviour on C-reactive protein

28 (CRP) measured in saliva. Two groups of women either reduced daily movement or increased

29 physical activity for 10 days. Salivary CRP increased by $31 \%$ in the sedentary group

$30 \quad\left(0.378 \pm 0.596\right.$ to $\left.0.487 \pm 0.793 \mu \mathrm{g} \cdot \mathrm{L}^{-1}\right)$ and decreased by $22 \%$ in the active group $(0.414 \pm 0.640$ to

$\left.310.259 \pm 0.284 \mu \mathrm{g} \cdot \mathrm{L}^{-1}\right)$. These results suggest short-term changes in daily movement behaviour can

32 affect salivary CRP, a marker of systemic inflammation.

33

34 Keywords: sedentary, physical activity, inflammation, $\mathrm{C}$ reactive protein, saliva, 35 


\section{INTRODUCTION}

Despite growing awareness of the potential health consequences of excessive sedentary time, the underlying physiological mechanisms remain unclear. Several nationally representative studies have shown a cross-sectional association between sedentary time and inflammatory markers such as C-reactive protein (CRP) (Healy et al. 2011; Carson et al. 2014).

CRP is a plasma protein and a component of the innate immune system (Du Clos 2000).

42 It is used in several areas of clinical practice, such as identifying acute infection and monitoring

43 the progression of some autoimmune diseases, such as rheumatoid arthritis (Plant et al. 2000).

44 Importantly, several epidemiological studies have shown that even when within a normal range,

45 higher serum CRP concentration is predictive of future cardiovascular events (Ridker et al.

46 2000).

Physical activity is inversely associated with CRP (Beavers et al. 2010), however, experimental studies of sedentary time and CRP are limited. One study reported an increase in serum CRP following 14 days of reduced activity in older adults (Breen et al. 2013). More longitudinal and intervention studies of CRP and movement behaviours are needed to confirm

51 cross-sectional evidence.

52 Currently, CRP is most commonly measured via blood sampling. Salivary diagnostics 53 represent a promising new technique that is cost effective, easy to use, and less invasive than

54 blood sampling (Malathi et al. 2014). Evidence supports an association between serum and 55 salivary CRP concentrations (Ouellet-Morin et al. 2011), and correlations have been established 56 between salivary CRP and subclinical atherosclerosis (Labat et al. 2013) and chronic obstructive 57 pulmonary disease (Patel et al. 2015). Salivary CRP has also been shown to increase acutely in 58 response to minimally invasive surgery (Foley et al. 2012). Together these data suggest salivary 
59 CRP is a valid measure of low grade inflammation. Thus, the purpose of this study was to

60 explore the effect of changes in daily movement behaviour on salivary CRP among healthy

61 women. We hypothesized that CRP would increase with reduced daily movement and decrease

62 with increased daily physical activity.

\section{MATERIALS AND METHODS}

64 Participants. Healthy females aged 40-60 years were recruited for this study. Inclusion

65 criteria were that participants be somewhat active or active, quantified by an average of 7,000 to

66 13,000 steps per day (Tudor-Locke et al. 2013), but not engaged in purposeful exercise.

67 Exclusion criteria included recent injury, acute illness or infection, obesity (BMI $\left.>30 \mathrm{~kg} \cdot \mathrm{m}^{-2}\right)$, or

68 any known chronic conditions. Participants provided written informed consent, and the study

69 was approved by the Institutional Human Subject Research Committee.

Preliminary Assessment. A 7-day preliminary assessment of movement behaviour was

71 completed; participants were blind to the step-count inclusion criteria and were told to maintain

72 their typical lifestyle. A Piezo Rx pedometer (Deep River, ON) was used to measure step count,

73 and an ActiGraph GT3X accelerometer (ActiGraph LLC, Pensacola, FL) was used to assess

74 sedentary time and physical activity. Baseline measures were then collected; eligible participants

75 were matched based on age and BMI then randomly assigned to one of two intervention groups,

76 sedentary or active.

77 Intervention Protocol. The intervention was 10 days. The active group increased their

78 individual daily step count by 3,000 steps, which is approximately equivalent to adding 30

$79 \mathrm{~min}$ /day of moderate intensity activity (Tudor-Locke et al. 2008). The sedentary group reduced

80 their daily step count to $<5000$ steps per day to mimic an inactive and sedentary lifestyle (Tudor-

81 Locke et al. 2008). Participants were directed to maintain their normal diet, report any illness or 
82 injury, and refrain from using any anti-inflammatory medications. (For study timeline see

83 Supplemental Figure 1).

84 Measurement of Movement Behaviour. During the intervention, participants wore a

85 pedometer to self-monitor their daily step count. Participants also wore an ActiGraph GT3X

86 accelerometer (ActiGraph, Pensacola, FL) on the hip. The ActiGraph recorded in 10-s periods,

87 and non-wear time was identified by 90 consecutive minutes of zero counts with a 2-min spike

88 tolerance (Choi et al. 2011). Sedentary time was defined as $<100$ counts $/$ min, and moderate to

89 vigorous physical activity (MVPA) was defined as $>1951$ counts/min (Freedson et al. 1998).

90 Accelerometer data were reduced using ActiLife version 6.1 software (ActiGraph, LLC,

91 Pensacola, Fla., USA).

92 Measurement of C-Reactive Protein. Saliva samples were collected at the end of the

93 preliminary assessment and at day 0 and Day 10 of the intervention. Serum CRP concentrations

$94>10 \mathrm{mg} / \mathrm{L}$ are often due to acute conditions and individuals with values above that should be

95 excluded (Pearson et al. 2003). Based on the conversion formula suggested by Ouellet-Morin et

96 al. (2011), we chose a salivary CRP cut-off, a priori, of $7 \mu \mathrm{g} / \mathrm{L}$.

97 All saliva samples were collected using the SalivaBio Oral Swab (Salimetrics, LLC., State

98 College, PA) according to manufacturer's instructions. Participants were asked to avoid eating,

99 smoking, or drinking for 1 hour prior to sample collection, consuming alcohol for 12 hours prior,

100 and to refrain from strenuous activity the day of sampling. CRP concentrations were assessed

101 using a high sensitivity enzyme-linked immunosorbent assay (ELISA) (Salimetrics, LLC., State

102 College, PA). Average intra- and inter- assay variability was $3.4 \%$ and $9.1 \%$, respectively.

103 Statistical Analysis. Statistical analyses were performed using IBM SPSS Statistics

104 (version 22.0; IBM Corp., Armonk, N.Y., USA). Salivary CRP concentrations did not meet the 
105 criteria of a normal distribution so logarithmic transformations were applied prior to analyses;

106 actual concentrations are presented. Preliminary comparisons between groups were performed

107 using independent sample t-tests (two-tailed). Difference in CRP from the preliminary

108 assessment to day 0 of the intervention were examined using a paired samples t-test. Difference

109 in CRP between groups and across time during the intervention were examined using a two-way

110 repeated measures ANOVA (group x time). Statistical significance was set at $p<0.05$. All values

111 are reported as mean $\pm \mathrm{SD}$.

\section{RESULTS}

113 Twenty women began the study; one participant in the sedentary group was injured during the

114 intervention and was omitted from analyses. In addition, preliminary CRP samples from 2

115 participants were not collected due to non-compliance with sampling guidelines. Table 1 shows

116 the baseline data.

117 Change in Movement Behaviour. During the intervention, all 19 participants wore the

118 accelerometer for 10 days, with an average wear time of $15.2 \pm 1.2$ hours per day. The sedentary

119 group significantly decreased their daily step count from $9,392 \pm 702$ to $3,854 \pm 147(\mathrm{p}<0.001)$ and

120 the active group increased from $8,772 \pm 427$ to $12,815 \pm 454$ steps per day $(\mathrm{p}<0.001)$. The

121 sedentary group increased their sedentary time by 69.8 minutes per day and significantly

122 decreased both their LPA and MVPA $(\mathrm{p}<0.001)$. The active group added 19 minutes of MVPA

123 per day $(\mathrm{p}<0.001)$ and sedentary time did not change significantly. During the intervention, the

124 sedentary group spent a greater proportion of waking time sedentary than the active group (85\%

125 versus $72 \%$, respectively) $(\mathrm{p}<0.001)$. Detailed activity profiles for each group can be seen in

126 Supplemental Table 2. 
127 C-reactive protein. From the end of the preliminary assessment period to the start of the

128 intervention (range of 9-21 days, $\mathrm{N}=17)$, CRP did not change significantly $(0.320 \pm 0.571$ to

$\left.129 \quad 0.297 \pm 0.435 \mu \mathrm{g} \cdot \mathrm{L}^{-1}, \mathrm{p}>0.05\right)$.

130 During the intervention $(\mathrm{N}=19)$ there was a significant crossover interaction $(\mathrm{p}<0.001)$ showing 131 that the two groups responded differently over time. In the sedentary group CRP increased in 132 seven of nine individuals with an average $31 \%$ increase $\left(0.378 \pm 0.596\right.$ to $\left.0.487 \pm 0.793 \mu \mathrm{g} \cdot \mathrm{L}^{-1}\right)$

133 and in the active group CRP decreased in all 10 participants with an average $22 \%$ decrease

$134\left(0.414 \pm 0.640\right.$ to $\left.0.259 \pm 0.284 \mu \mathrm{g} \cdot \mathrm{L}^{-1}\right)$. The individual relative change and individual absolute 135 concentration change can be seen in Supplemental Figure 2.

\section{DISCUSSION}

137 The present study adds experimental evidence to the increasing number of cross-sectional 138 studies that have shown an association between movement behaviour and CRP (Carson et al. 139 2014; Healy et al. 2011). Limited experimental studies have been conducted, most using serum 140 CRP and clinical populations. Breen et al. (2013) showed a 12\% increase in serum CRP after 2 141 weeks of reduced activity in elderly individuals. The only other study to use salivary CRP to 142 examine activity effects reported a 54\% decrease in in CRP after 8 weeks of exercise in male 143 cardiac patients (Jamshidpour et al. 2013). This is the first study to show that salivary CRP is 144 sensitive to changes in daily movement behaviour in healthy women.

145 Quantifying CRP, as well as other biochemicals, via saliva is a useful advancement in 146 bioanalysis techniques for researchers who are often constrained by budget, personnel, and 147 willingness of volunteer participants. Like blood, saliva is a dynamic and complex fluid that 148 contains an array of enzymes, hormones, antibodies, and cytokines, that can reflect the 149 physiologic state of the body (Aps and Martens 2005). CRP likely enters the oral cavity via the 
150 gingival crevicular fluid, which can be either a transudate or inflammatory exudate of serum, that 151 mixes to becomes a constituent of whole saliva (Lamster and Ahlo 2007).

152 The mechanisms by which movement influences CRP are unclear. (Hamilton et al. 2004).

153 Previous studies have suggested that physical activity reduces inflammation by decreasing body

154 fat (Oberbach et al. 2006), increasing insulin sensitivity (Oberbach et al. 2006), improving

155 endothelial function (Taddei et al. 2000), or by the acute release of anti-inflammatory cytokines

156 with each bout of exercise (Gleeson et al. 2011). The increase in CRP in the sedentary group

157 could also be due to a number of factors, such as inhibition of anti-inflammatory myokines due

158 to reduced muscular contractions (Henson et al. 2013). The sedentary group significantly

159 increased sedentary time, but also decreased their MVPA. Therefore, it is possible that the

160 removal of daily MVPA, rather than the increased sedentary time, caused the increase in CRP; it

161 is also plausible that both changes contributed.

162 This exploratory study does have limitations. The sample size is small, and we did not

163 control for menstrual status. Also, dietary records were not used and it is presumed that

164 participants adhered to instructions about diet and medication use during the intervention. Due to

165 matching and randomization procedures, the time between the end of preliminary assessment and

166 start of the intervention varied across participants (9-21 days) and this limits the interpretation of

167 the CRP concentrations between these two samples.

168 In conclusion, 10 days of altered movement behaviour resulted in significant changes in

169 salivary CRP, consistent with cross-sectional evidence of associations between sedentary time

170 and serum CRP. Inflammation may be a potential link between movement behaviour and health

171 outcomes in healthy middle-aged women. The use of salivary measures of CRP and other 
172 inflammatory markers should be considered for future investigations as this may allow

173 researchers to explore and develop this field more quickly.

\section{Conflict of interest statement}

175 The authors have no conflicts of interest to declare.

176

177 
178

179

180

181

182

183

184

185

186

187

188

189

190

191

192

193

194

195

196

197

198

\section{References}

Aps, J.K.M., and Martens, L.C. 2005. Review: The physiology of saliva and transfer of drugs into saliva. Forensic Sci. Int. 150(2-3): 119-131. doi:10.1016/j.forsciint.2004.10.026

Beavers, K.M., Brinkley, T.E., and Nicklas, B.J. 2010. Effect of exercise training on chronic inflammation. Clin. Chim. Acta. 411(11-12): 785-793. doi:10.1016/j.cca.2010.02.069

Breen, L., Stokes, K.A., Churchward-Venne, T.A., Moore, D.R., Baker, S.K., Smith, K. et al. 2013. Two weeks of reduced activity decreases leg lean mass and induces "anabolic resistance" of myofibrillar protein synthesis in healthy elderly. J. Clin. Endocrinol. Metab. 98(6): 2604-2612. doi:10.1210/jc.2013-1502

Carson, V., Wong, S.L., Winkler, E., Healy, G.N., Colley, R.C., and Tremblay, M.S. 2014. Patterns of sedentary time and cardiometabolic risk among canadian adults. Prev. Med. 65: 23-27. doi:10.1016/j.ypmed.2014.04.005

Choi, L., Liu, Z., Matthews, C.E., and Buchowski, M.S. 2011. Validation of accelerometer wear and nonwear time classification algorithm. Med. Sci. Sports Exerc. 43(2): 357-364. doi:10.1249/MSS.0b013e3181ed61a3

Du Clos, T.W. 2000. Function of c-reactive protein. Ann. Med. 32(4): 274-278. doi: $10.3109 / 07853890009011772$

Foley, J.D., Sneed, J.D., Steinhubl, S.R., Kolasa, J., Ebersole, J.L., Lin, Y.S. et al. 2012. Oral fluids that detect cardiovascular disease biomarkers. Oral Surgery Oral Medicine Oral Pathology Oral Radiology. 114(2): 207-214. doi:10.1016/j.oooo.2012.03.003 
Freedson, P.S., Melanson, E., and Sirard, J. 1998. Calibration of the computer science and applications, inc. Accelerometer. Med. Sci. Sports Exerc. 30(5): 777-781. doi:10.1097/00005768-199805000-00021

Gleeson, M., Bishop, N.C., Stensel, D.J., Lindley, M.R., Mastana, S.S., and Nimmo, M.A. 2011. The anti-inflammatory effects of exercise: Mechanisms and implications for the prevention and treatment of disease. Nat. Rev. Immunol. 11(9): 607-615. doi:doi:10.1038/nri3041

Hamilton, M.T., Hamilton, D.G., and Zderic, T.W. 2004. Exercise physiology versus inactivity physiology: An essential concept for understanding lipoprotein lipase regulation. Exerc.

Healy, G.N., Matthews, C.E., Dunstan, D.W., Winkler, E.A., and Owen, N. 2011. Sedentary time Sport Sci. Rev. 32(4): 161-166. and cardio-metabolic biomarkers in us adults: Nhanes 2003-06. Eur. Heart J. 32(5): 590597. doi:10.1093/eurheartj/ehq451

215 Jamshidpour, B., Moghadam, B.A., Vasaghi-Gharamaleki, B., Mirzaii-Dizgah, I., and Nejatian, 216 M. 2013. The effects of phase iii cardiac rehabilitation in serum and salivary hs-crp and 217 anthropometric measurements in patients with coronary artery disease. J. Contemp. Dent. Pract. 14(5): 819-824. 
Labat, C., Temmar, M., Nagy, E., Bean, K., Brink, C., Benetos, A. et al. 2013. Inflammatory mediators in saliva associated with arterial stiffness and subclinical atherosclerosis. J. Hypertens. 31(11): 2251-2258. doi:10.1097/HJH.0b013e328363dccc

222 223

Lamster, I.B., and Ahlo, J.K. 2007. Analysis of gingival crevicular fluid as applied to the diagnosis of oral and systemic diseases. Ann. N. Y. Acad. Sci. 1098: 216-229. doi:10.1196/annals.1384.027

Malathi, N., Mythili, S., and Vasanthi, H.R. 2014. Salivary diagnostics: A brief review. ISRN Dentistry. 2014: 1-8.

Oberbach, A., Tonjes, A., Kloting, N., Fasshauer, M., Kratzsch, J., Busse, M.W. et al. 2006. Effect of a 4 week physical training program on plasma concentrations of inflammatory markers in patients with abnormal glucose tolerance. Eur. J. Endocrinol. 154(4): 577-585. doi:10.1530/eje.1.02127

Ouellet-Morin, I., Danese, A., Williams, B., and Arseneault, L. 2011. Validation of a highsensitivity assay for c-reactive protein in human saliva. Brain Behav. Immun. 25(4): 640646. doi:10.1016/j.bbi.2010.12.020

Patel, N., Belcher, J., Thorpe, G., Forsyth, N.R., and Spiteri, M.A. 2015. Measurement of creactive protein, procalcitonin and neutrophil elastase in saliva of copd patients and healthy controls: Correlation to self-reported wellbeing parameters. Respir. Res. 16. doi:10.1186/s12931-015-0219-1

Pearson, T.A., Mensah, G.A., Alexander, R.W., Anderson, J.L., Cannon, R.O., Criqui, M. et al. 2003. Markers of inflammation and cardiovascular disease application to clinical and 
public health practice: A statement for healthcare professionals from the centers for disease control and prevention and the american heart association. Circulation. 107(3):

Plant, M.J., Williams, A.L., O'Sullivan, M.M., Lewis, P.A., Coles, E.C., and Jessop, J.D. 2000. Relationship between time-integrated c-reactive protein levels and radiologic progression in patients with rheumatoid arthritis. Arthritis Rheumatol. 43(7): 1473-1477. 499-511. doi:10.1161/01.cir.0000052939.59093.45

Ridker, P.M., Hennekens, C.H., Buring, J.E., and Rifai, N. 2000. C-reactive protein and other markers of inflammation in the prediction of cardiovascular disease in women. N. Engl.

Taddei, S., Galetta, F., Virdis, A., Ghiadoni, L., Salvetti, G., Franzoni, F. et al. 2000. Physical J. Med. 342(12): 836-843. doi:10.1056/NEJM200003233421202 activity prevents age-related impairment in nitric oxide availability in elderly athletes. Circulation. 101(25): 2896-2901. lifestyle index: <5000 steps/day. Appl. Physiol. Nutr. Metab. 38(2): 100-114. are enough?". Med. Sci. Sports. Exerc. 40(7 Suppl): S537-543. doi:10.1249/MSS.0b013e31817c7133 
Table 1. Anthropometric, physiological, and behavioral characteristics of sedentary and active groups during the preliminary assessment.

\begin{tabular}{llll}
\hline \hline & $\begin{array}{l}\text { Sedentary } \\
(\mathrm{n}=9)\end{array}$ & $\begin{array}{l}\text { Active } \\
(\mathrm{n}=10)\end{array}$ & $\mathrm{P}$ \\
\hline Age $(\mathrm{yr})$ & $49.6 \pm 5.6$ & $49.9 \pm 5.2$ & 0.891 \\
Height $(\mathrm{m})$ & $1.64 \pm 5.3$ & $1.66 \pm 5.2$ & 0.413 \\
Weight $(\mathrm{kg})$ & $76.5 \pm 7.7$ & $73.6 \pm 10.5$ & 0.518 \\
BMI $\left(\mathrm{kg} \cdot \mathrm{m}^{-2}\right)$ & $28.4 \pm 3.5$ & $26.6 \pm 3.7$ & 0.296 \\
WC $(\mathrm{cm})$ & $90.7 \pm 10.4$ & $87.0 \pm 8.3$ & 0.399 \\
CRP $(\mu \mathrm{g} / \mathrm{L})$ & $0.45 \pm 0.78$ & $0.53 \pm 1.04$ & 0.857 \\
Steps $(\mathrm{steps} /$ day $)$ & $9392 \pm 2106$ & $8772 \pm 1350$ & 0.451 \\
\hline
\end{tabular}

All values are presented as mean $\pm \mathrm{SD}$ 